\title{
Study on the Iterative Compensation Method for Continuous Varying Curvature Free Bending
}

\author{
Ruixue Zhai $\mathbb{D}^{1,2}$ Pengcheng Fu $\mathbb{D}^{1,2}$ Rui Ma $\mathbb{D}^{1,2}$ Jun Zhao, ${ }^{1,2}$ and Cuiyun Ge \\ ${ }^{1}$ Key Laboratory of Advanced Forging and Stamping Technology and Science (Yanshan University), \\ Ministry of Education of China, Qinhuangdao 066004, China \\ ${ }^{2}$ College of Mechanical Engineering, Yanshan University, Qinhuangdao 066004, China \\ ${ }^{3}$ Capital Engineering and Research Incorporation Ltd, Beijing 100176, China \\ Correspondence should be addressed to Rui Ma; mar@ysu.edu.cn
}

Received 4 June 2021; Revised 7 September 2021; Accepted 7 October 2021; Published 5 November 2021

Academic Editor: Rossana Dimitri

Copyright (c) 2021 Ruixue Zhai et al. This is an open access article distributed under the Creative Commons Attribution License, which permits unrestricted use, distribution, and reproduction in any medium, provided the original work is properly cited.

\begin{abstract}
The parts formed by the bending process not only have high precision of appearance dimension but also have good performance. In recent years, enterprises have put forward higher requirements for the forming process and product quality. Therefore, a new method for iterative compensation of bending springback with certain generality is proposed for continuous curvature bending. The purpose of this study is to take curvature as an iterative parameter and make the shape size reach the expected value through the finite compensation. On the basis of establishing this iterative compensation mechanism, the convergence of curvature iteration in the general free bending process is proved. The reliability of the proposed iterative compensation method in the bending process engineering application is verified by combining simulation with experiment. The two materials of 304 stainless steel and ST12 cold rolled steel were studied, and the two-dimensional plane stress-strain model Abaqus cantilever beam was established by using finite element software. The bending forming simulation was carried out based on the above iterative compensation mechanism. Finally, through the bending experiments of four models, the feasibility of the iterative compensation mechanism of curvature in the continuous curvature plane bending process is verified, and different models are selected to clarify that the method has the characteristics of generality, that is, it will greatly improve the flexibility of the bending process in industrial applications without the limitation of material types and mechanical models.
\end{abstract}

\section{Introduction}

The bending forming process of sheet metal refers to the method of forming the sheet metal under the action of applying external force by using the elastic-plastic characteristics of the material. Metal parts must rebound during elastoplastic forming. The outer fiber is subjected to tensile stress, while the inner fiber is subjected to compressive stress during bending. After unloading, the workpiece is restored by elasticity, the outer fiber is shortened, and the inner fiber is elongated; thus, the rebound phenomenon occurs [1]. Over the years, many scholars at home and abroad [2-4] have made some achievements in the study of springback problem. The control of springback is mostly by the die compensation method [5] or process control method. The research means include single or interactive use of theoretical analysis [6,7], finite element simulation $[8,9]$, and experimental verification $[10,11]$.The problem of plane bending with variable curvatures is a hot topic at present. The complex problem of plane bending with continuous curvature can be solved by modifying the process parameters by the iterative compensation method. In this study, it is also from the iterative point of view in the theoretical environment of the analysis and calculation [12, 13].

Abaqus is a common software for analyzing mechanical systems at present, which can analyze complex solid mechanics and structural mechanics problems and deal with highly nonlinear problems. Because of the reliability of its simulation complex system, it has been widely used in industry and research in various countries. A finite element 
software Abaqus is used to simulate the bending springback $[14,15]$.

After proving the theory at the theoretical level, this study will also verify the feasibility of the curvature iterative compensation mechanism based on four mathematical models from the perspective of experimental analysis. Finally, the experimental results are compared with the theoretical and simulation results to verify the reliability of the iterative mechanism in the continuous curvature plane bending rebound problem.

\section{The Theory of Iterative Compensation for Continuous Curvature Plane Bending}

2.1. Brief Introduction of Iterative Compensation Mechanism. The iterative compensation mechanism is a method to obtain the target value by modifying the iterative parameters in a finite order. This method is equivalent to the mold repair process in engineering, but it is more directional, faster, and more efficient than the mold repair technology, which provides theoretical guidance for the manufacture of the die needed for bending forming.

In mathematics, the successive approximation method of equation root finding is the application of the iterative compensation mechanism. The application of this method is described in detail in numerical analysis.

For the general springback problem, the equation is constructed by selecting the appropriate iterative parameters $y=f(x)$, where $x$ represents the pre-rebound values of selected research parameters, and $y$ denotes the rebound value of the parameter. Curvilinear function $y=f(x)$ is the elastic complex equation of selected parameters and must comply $f^{\prime}(x)<1$. The method of iterative mechanism compensation is effective.

The general steps of the iterative compensation method in springback problem are as follows:

(1) Determination of the elastic complex equation such as $y=f(x)$. Then, the convergence of each process parameter is verified. Select the appropriate iteration parameter and take the target value as the initial value of the iteration parameter.

(2) The rebound value which $x_{1}=f\left(x_{0}\right)$ is obtained by using the existing elastic complex equation

(3) Check $\left|x_{1}-x_{0}\right|$ whether to meet the accuracy requirements. If satisfied, the iterative compensation process ends. If not, superimposed $\left|x_{1}-x_{0}\right|$ as compensation on $x_{0}$. Return to the previous step and continue the iteration until accuracy is met.

(4) Output iteration in which $x_{1}$ results as final result

2.2. Convergence Criterion of Springback Iterative Compensation and Parameter Determination. Let $x$ be the value of the control parameter before springback, and $f(x)$ is the function expression between the control parameters before and after springback; then, the springback value $\Delta(x)$ can be expressed as

$$
\Delta(x)=x-f(x)
$$

According to the above theoretical basis, if $\Delta(x)$ is a monotonically increasing function, that is, $\Delta^{\prime}(x)>0$, then there is

$$
f^{\prime}(x)<1
$$

The purpose of springback control is to determine a parameter value $a$ that changes to $a_{\rho}$ after springback, that is, solving the equation

$$
f(x)-a_{\rho}=0 .
$$

For this reason, the simple iteration method is introduced. According to the thought of solving equation, the iterative equation is constructed as

$$
x=x+a_{\rho}-f(x)=\varphi(x) .
$$

Combined with equation (2), it can be deduced that

$$
\left|\varphi^{\prime}(x)\right|<1 \text {. }
$$

The iterative equation equation (4) is convergent by the local convergence theorem of the simple iterative method, that is, where $x^{*}$ satisfies $x^{*}=\varphi\left(x^{*}\right)$. Thus, as shown in Figure 1 , the initial value is chosen as $a_{\rho}$; then, the iterative sequence is obtained according to the iterative equation as

$$
\begin{aligned}
x_{0} & =a_{\rho} \\
x_{1} & =x_{0}+a_{\rho}-f\left(x_{0}\right) \\
x_{2} & =x_{1}+a_{\rho}-f\left(x_{1}\right) \\
& \vdots \\
x_{i} & =x_{i-1}+a_{\rho}-f\left(x_{i-1}\right) \\
& \vdots \\
x_{k} & =x_{k-1}+a_{\rho}-f\left(x_{k-1}\right) .
\end{aligned}
$$

For a preset error $\varepsilon$, when $\left|x_{k}-x_{k-1}\right| \leq \varepsilon$, it is recognised that $x^{*} \approx x_{k}$; then, $f\left(x^{*}\right)-a_{\rho}=0$ is the request.

For the springback control problem, if the relation function $y=f(x)$ of the parameters before and after the springback satisfies $f^{\prime}(x)<1$, the iterative compensation method can be used to control the parameters to converge to the target value. Therefore, for the compensation calculation of the springback problem, equation (2) can be used as the convergence criterion of the selected parameters, and this convergence method is applicable to parameters that are smaller after springback.

When the curvature satisfies, $K>2 \sigma_{s} / E$, derogation $K^{\prime}$ to $K$ :

$$
\frac{\mathrm{d} K^{\prime}}{\mathrm{d} K}=\left(1-\frac{D}{E}\right) \cdot\left[\frac{1-\left(\sigma_{s} / E\right)^{3}}{K^{3}}\right]
$$

$D$ represents the tangent modulus, and $E$ represents the young's modulus.

We can sort it out as 


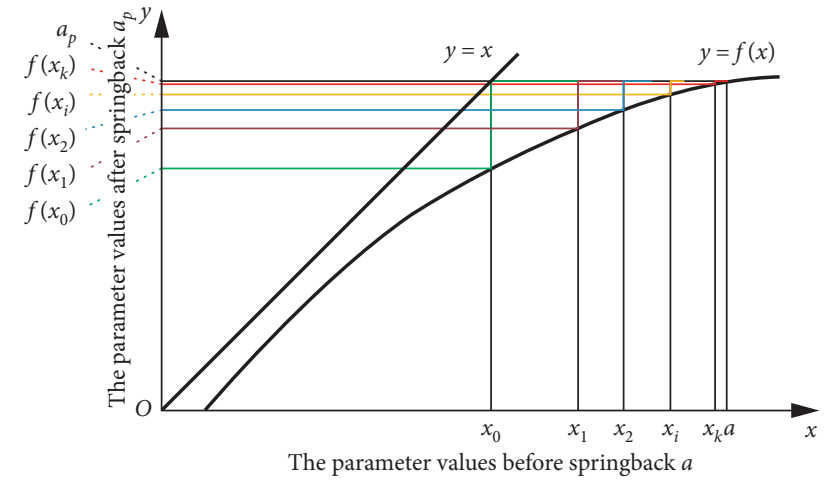

Figure 1: Parameter relationship before and after springback based on the simple iteration method.

$$
0<\frac{\mathrm{d} K^{\prime}}{\mathrm{d} K}<1-\frac{D}{E}<1
$$

From this, we can see that the curvature can be used as an iterative parameter of the iterative compensation mechanism in the free bending process of sheet metal.

2.3. A Plane Bending Elastic Complex Model. The plate model is in accordance with the following basic assumptions and models: flat section hypothesis, unidirectional stress state hypothesis, conventional elastic-plastic material hypothesis, small curvature hypothesis, and linear distribution of strain in bending deformation section; most importantly, it fits the bilinear hardening model:

$$
\sigma= \begin{cases}E \cdot \varepsilon, & 0 \leq \varepsilon \leq \frac{\sigma_{s}}{E}, \\ D \cdot \varepsilon+\sigma_{s} \cdot\left(1-\frac{D}{E}\right), & \varepsilon>\frac{\sigma_{s}}{E} .\end{cases}
$$

The tangential strain corresponding to the yield limit is

$$
\varepsilon_{s}=\frac{\sigma_{s}}{E} .
$$

When the inner and outer surfaces begin to yield, the radius of the neutral layer can be expressed as

$$
\rho_{s}=\frac{(1 / 2)}{\varepsilon_{s}}=\frac{t E}{2 \varepsilon_{s}}
$$

At this point, the curvature of the neutral layer is expressed as

$$
K_{s}=\frac{1}{\rho_{s}}=\frac{2 \sigma_{s}}{\mathrm{tE}} .
$$

Therefore, the condition of plastic deformation of sheet metal during free bending is that the radius of neutral layer or its curvature value satisfies the following relation:

$$
\begin{aligned}
& 0<S_{r} \leq \rho_{s}=\frac{t E}{2 \sigma_{s}}, \\
& S_{k} \geq K_{s}=\frac{2 \sigma_{s}}{t E} .
\end{aligned}
$$

The moment formed by tangential stress is expressed as

$$
M=2 \int_{0}^{1 / 2} \sigma \cdot y \mathrm{~d} A .
$$

The linear distribution of the strain on the bending deformation section shows that $y=\rho \cdot \varepsilon, \mathrm{d} A=b \cdot \rho \cdot \mathrm{d} \varepsilon$. For the bilinear hardening model, the moment expression is

$$
M=E \cdot I \cdot \frac{1}{\rho} \cdot\left\{\frac{\sigma_{s}}{E} \cdot\left(1-\frac{D}{E} \cdot\left[3-4 \cdot\left(\frac{\sigma_{s}}{E}\right)^{2} \cdot\left(\frac{\rho}{t}\right)^{2}\right] \cdot \frac{\rho}{t}+\frac{D}{E}\right\}\right.
$$

According to the classical unloading theory, the amount of curvature rebound is the difference of curvature before and after rebound:

$$
\Delta K=K-K^{\prime}=\frac{1}{\rho}-\frac{1}{\rho^{\prime}}=\frac{M}{E I} .
$$

Then, we can get the expression of the curvature of springback about each parameter as follows:

$$
K^{\prime}=\left(1-\frac{D}{E}\right) \cdot\left[K+\frac{4}{k^{2} t^{3}} \cdot\left(\frac{\sigma_{s}}{E}\right)^{3}\right]-\frac{3 \sigma_{3}}{E t}\left(1-\frac{D}{E}\right),\left(k>\frac{2 \sigma_{s}}{E}\right)
$$


So, the elastic complex equation of the curvature of the neutral layer of sheet metal during free bending is as follows:

$$
K^{\prime}= \begin{cases}0, & \left(K \leq \frac{2 \sigma_{s}}{t E}\right), \\ \left(1-\frac{D}{E}\right) \cdot\left[K+\frac{4}{k^{2} t^{3}} \cdot\left(\frac{\sigma_{s}}{E}\right)^{3}\right]-\frac{3 \sigma_{3}}{E t}\left(1-\frac{D}{E}\right), & \left(K>\frac{2 \sigma_{s}}{E}\right) .\end{cases}
$$

2.4. Mathematical Basis of the Iterative Compensation Mechanism for Variable Curvature. The function corresponding to setting the target shape of the sheet metal neutral layer is expressed as $y_{0}=f(x)$; after the rebound, the neutral layer curve type of sheet metal remains unchanged, and the corresponding function is expressed as $y_{0 s}=f_{s}(x)$. Use the iterative compensation mechanism to correct the curvature of the surface curve after the equation expressed as $y_{1}=f_{1}(x)$.

The length of the neutral layer of the sheet metal remains unchanged when elastic-plastic deformation occurs. Therefore, in the study of the bending springback process of variable curvature, the coordinates of the data points after springback and compensation can be obtained by the arc length limiting condition, and the corresponding curvature can be obtained by the elastic complex equation and compensation method. The coordinate and curvature values of each data point are determined by arc length and curvature limitation conditions, so that the function equation of springback and correction compensation can be solved.

2.4.1. Arc Length and Its Limiting Conditions. When the object of study is curve, arc length is often introduced as parameter. For the same data point, node- $i, i=0,1,2 \ldots n$. If the fixed point is located in $x=\eta$ for analysis, assume that the corresponding coordinates before $x$ rebound are $x_{0-i}$ and the corresponding coordinates after rebound are $x_{0 s-i}$. After iteratively compensating the curvature, the corresponding coordinates of the data point are $x_{1-i}$. At this point, in the springback before and after the compensation curve, arc lengths $x=\eta$ to node- $i$ at fixed points can be expressed as follows:

$$
\left\{\begin{array}{l}
L_{0}=\int_{\eta}^{x_{0-i}} \sqrt{1+\left(y_{0}^{\prime}\right)^{2}(x)} d(x) \\
L_{0 s}=\int_{\eta}^{x_{0 s-i}} \sqrt{1+\left(y_{0 s}^{\prime}\right)^{2}(x)} d(x) \\
L_{1}=\int_{\eta}^{x_{1-i}} \sqrt{1+\left(y_{1}^{\prime}\right)^{2}(x)} d(x)
\end{array}\right.
$$

We can know $L_{0}=L_{0 s}=L_{1}$. In order to reduce the error, the equivalent relation is taken as springback calculation: $L_{0}=L_{s}$ and iterative compensation calculation: $L_{0}=L_{1}$.

2.4.2. Limitation of Curvature. For the same data point, node-i, $i=0,1,2 \ldots n$. If the fixed point is located in $x=\eta$ for analysis, assume that the corresponding coordinates before $x$ rebound are $x_{0-i}$, and the corresponding coordinates after rebound are $x_{0 s-i}$. After iteratively compensating the curvature, the corresponding coordinates of the data point are $x_{1-i}$. Hence, the curvature node- $i$ is expressed as

$$
\begin{gathered}
K_{\text {Node }-i}=K_{x 0-i}=\frac{y_{0-i}^{\prime \prime}}{\left[1+\left(y_{x 0-i}^{\prime}\right)^{2}\right]^{3 / 2}}, \\
K_{\text {Node }-i}^{\prime}=K_{x 0 s-i}=\frac{y_{0 s-i}^{\prime \prime}}{\left[1+\left(y_{x 0 s-i}^{\prime}\right)^{2}\right]^{3 / 2}} .
\end{gathered}
$$

For the elastic model of continuous curvature sheet metal bending freely, it is considered that the curvature relation of each data point of the continuous curvature equation satisfies the elastic complex equation of the curvature of the neutral layer (formula (11)) when the uniform curvature plane bends, and the following formula can be obtained by substituting formulae (13) and (14) into (11):

$$
K_{\text {Node }-i}^{\prime}=\left\{\begin{array}{lr}
0, & \left(K_{\text {Node }-i} \leq \frac{2 \sigma_{s}}{t E}\right), \\
\left(1-\frac{D}{E}\right) \cdot\left[K_{\text {Node }-i}+\frac{4}{K_{\text {Node }-i}^{2} t^{3}} \cdot\left(\frac{\sigma_{s}}{E}\right)^{3}\right]-\frac{3 \sigma_{3}}{E t}\left(1-\frac{D}{E}\right), & \left(K_{\text {Node }-i}>\frac{2 \sigma_{s}}{E}\right) .
\end{array}\right.
$$

The elastic complex equation of curvature on the neutral layer is abbreviated as $K^{\prime}=f_{k}(K)$. At this point, the following formula is established for limiting curvature: 


$$
\left\{\begin{array}{l}
K_{\text {Node }-i}=K_{x 0-i}=\frac{y_{0-i}^{\prime \prime}}{\left[1+\left(y_{x 0-i}^{\prime}\right)^{2}\right]^{3 / 2}} \\
K_{\text {Node }-i}^{\prime}=K_{x 0 s-i}=\frac{y_{0 s-i}^{\prime \prime}}{\left[1+\left(y_{x 0 s-i}^{\prime}\right)^{2}\right]^{3 / 2}} \\
K_{\text {Node }-i}^{\prime}=f_{k}\left(K_{\text {Node }-i}\right)
\end{array}\right.
$$

For every data point on a quadratic polynomial function, initial $x$ coordinates are specified by us and remain consistent with the selection in the arc length limiting conditions. Finally, we associate formulae (12) and (16) at each point of the study, and the coordinate values after springback and iterative compensation and the unknown coefficients in the curve equation are obtained by using the undetermined coefficient method.

\subsection{Software Foundation of the Iterative Compensation} Mechanism for Variable Curvature. Mathcad used in this study is a mathematical general class software which integrates mathematical programming and graphic drawing. It comes with a variety of language systems, simple algorithm, but extremely powerful computing. Mathcad has its own fixed solution module, different solution blocks can be called in the solution, and the corresponding limiting conditions can be substituted into the actual problem to solve the corresponding solution. Given find solution block is the main solution module used in this study.

\section{Theoretical Application of the Iterative Compensation Mechanism in Continuous Curvature Bending}

3.1. Expression of the Compensation Iterative Mechanism in Continuous Curvature Plane Bending. According to the above theoretical basis, the following conclusions can be obtained: the curvature can be used as an iterative compensation parameter in the free bending process of sheet metal, and there is a clear expression of elastic complex equation. Therefore, the iterative compensation mechanism can be applied in the planar bending process. Figure 2 shows the flowchart and application steps of the curvature iterative compensation mechanism in the continuous curvature plane bending process.

The application steps of the iterative compensation mechanism for curvature in continuous curvature plane bending are as follows:

(1)

(i) The elastic complex equation for determining the curvature of the neutral layer: $K^{\prime}=f_{k}(K)$

(ii) The curvature is convergent and can be used as an iterative compensation parameter

(iii) A target $K_{0}$ is taken as the initial value of the iteration parameter
(2) The curvature value after springback is obtained by using the curvature elastic complex equation: $K_{i+1}=f_{k}\left(K_{i}\right)$

(3) Check whether $\left|K_{0}-K_{i+1}\right|$ meets the accuracy requirements. If satisfied, the iterative compensation process ends; if not, to add $\left|K_{0}-K_{i+1}\right|$ as compensation to $K_{i}$ as a new $K_{i}$ value, return to the previous step and continue to iterate until the error between the curvature value and the target value after springback meets the precision requirement.

(4) Output the iterative result $K_{i}$ as the final result

In this study, we use four mathematical models (Table 1) to study on the springback of continuous curvature plane bending. These four mathematical models are expressed by a combination of two material models (ST12 and 304) and two function types (quadratic polynomial and exponential function).

3.2. Theoretical Results of Four Models. This subsection will analyze the results of the first model in detail, the remaining three are only shown as results, and the calculation process will not be repeated.

3.2.1. Quadric Function-ST12 Iteration Results. Made of $2 \mathrm{~mm}$ thick ST12 cold rolled steel, determine the function equation of the objective curve as $y=0.001 x^{2}+0.002 x$. For the first iteration operation, the target curve is selected as the iterative loading curve, and selected 15 data points of node- $i$ are used and studied. There is a difference between the curvature of the actual rebound curve and the curvature of the target, written $\Delta$. The first result of the ST12 model which is a quadratic function is given in Table 2 .

Obviously, after the first loading, the curvature of each data point decreases after rebound, and $\Delta$ values are in the neighborhood of 0.0012 . On account, MSE's value is

$$
\mathrm{MSE}_{1}=\frac{1}{15} \cdot \sum_{i=0}^{14}\left(\Delta_{i}\right)^{2}=1.44 \times 10^{-6} \text {. }
$$

Actually, in a program that uses software Mathcad for theoretical calculations, it will compensate the curvature value as long as the $\Delta$ value is greater than the set curvature allowable error. The compensation curve equation is obtained by fitting the compensation curvature data for the second iteration operation. The equation of compensation curve obtained by fitting is $y=0.0017 x^{2}+0.0033 x+0.0007$. The continuous curve of the equation is used to operate based on the curvature iterative compensation mechanism established in this study. Applying Mathcad software programming solution, the results obtained are given in Table 3 .

For the second iteration operation using the compensation curve, the curvature values of each point after rebound become larger than the first time, and the curvature error decreases, and the $\Delta$ value is in the neighborhood of 0 . Calculated, the MSE value of curvature is 


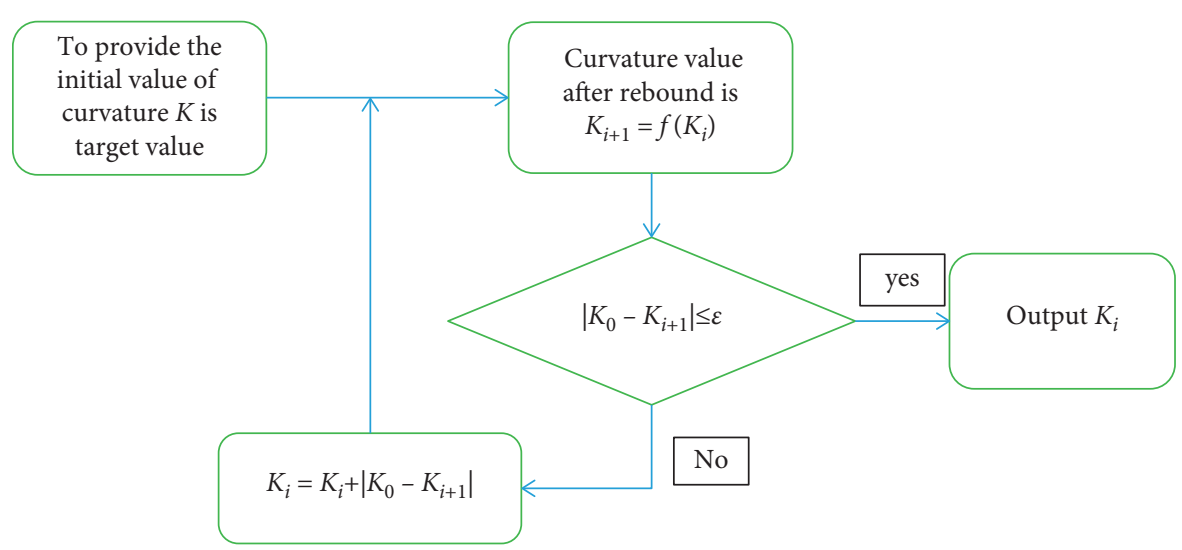

FIGURE 2: Flowchart of the curvature iteration method in the continuous curvature plane bending process.

TABLE 1: Four models of theoretical research.

\begin{tabular}{lcr}
\hline Model & Materials and brands & Objective shape function \\
\hline Polys-ST12 & Cold rolled steel ST12 & $y=0.001 x^{2}+0.002 x$ \\
Exp-ST12 & Cold rolled steel ST12 & $y=e^{0.03 x}$ \\
Polys-304 & Stainless steel 304 & $y=0.001 x^{2}+0.002 x$ \\
Exp-304 & Stainless steel 304 & $y=e^{0.02 x}$ \\
\hline
\end{tabular}

Polys, quadratic polynomial; Exp, exponential function.

TABLE 2: Calculation results of data points for second compensation of polynomial function.

\begin{tabular}{lccc}
\hline Data points $x$ subscripts & Curvature before rebound $/ 10^{-6}$ & Curvature after rebound/10 & Error amount $\Delta / 10^{-6}$ \\
\hline 0 & 2000 & 784 & 1216 \\
1 & 2000 & 783 & 1216 \\
2 & 1999 & 782 & 1216 \\
3 & 1997 & 787 & 1216 \\
4 & 1995 & 779 & 1216 \\
5 & 1989 & 773 & 1215 \\
6 & 1980 & 766 & 1214 \\
7 & 1975 & 761 & 1214 \\
8 & 1969 & 756 & 1214 \\
9 & 1956 & 744 & 1212 \\
10 & 1949 & 737 & 1212 \\
11 & 1933 & 722 & 1210 \\
12 & 1914 & 706 & 1208 \\
13 & 1905 & 697 & 1208 \\
14 & 1894 & 688 & 1207 \\
\hline
\end{tabular}

TABLE 3: Calculation results of data points for second compensation of polynomial function.

\begin{tabular}{lccc}
\hline Data points $x$ subscripts & Curvature before rebound $/ 10^{-6}$ & Curvature after rebound/10 & Error amount $\Delta / 10^{-6}$ \\
\hline 0 & 3216 & 2125 & -125 \\
1 & 3216 & 2123 & -123 \\
2 & 3215 & 2118 & -119 \\
3 & 3213 & 2110 & -113 \\
4 & 3211 & 2099 & -104 \\
5 & 3204 & 2070 & -81 \\
6 & 3194 & 2030 & -50 \\
7 & 3189 & 2006 & -31 \\
8 & 3183 & 1980 & -11 \\
9 & 3168 & 1922 & 34 \\
10 & 3161 & 1889 & 60 \\
11 & 3143 & 1818 & 115 \\
12 & 3122 & 1741 & 173 \\
13 & 3113 & 1700 & 205 \\
14 & 3101 & 1658 & 236 \\
\hline
\end{tabular}




$$
\mathrm{MSE}_{2}=\frac{1}{15} \cdot \sum_{i=0}^{14}\left(\Delta_{i}\right)^{2}=1.25 \times 10^{-8}
$$

The mean square of the residuals is 0 in the range of $10^{-6}$, which meets the required accuracy requirements. At this point, the curvature data after springback fitting are the type of quadratic function curve. The fitting parameters are $a=0.001, b=0.002$, and $c=0$. At this point, the iteration ends, and the continuous curvature free bending forming parts are obtained by correcting the curvature K.

\subsubsection{Exponential Function-ST12 Iteration Results.} Exponential function- iterative results of the ST12 model are shown in Figure 3; the expected value can be reached after two iterative compensations for curvature.

In the first iteration model, curvature error $\Delta$ is in the left neighborhood of 0.0013 . After calculation, MSE of the residual curvature is

$$
\mathrm{MSE}_{1}=\frac{1}{15} \cdot \sum_{i=0}^{14}\left(\Delta_{i}\right)^{2}=1.51 \times 10^{-6} .
$$

For the second iteration, the equation of compensation curve is expressed as $y=e^{0.032 x}$. After rebound, the curvature error decreases and the $\Delta$ value is in the neighborhood of 0 . After calculation, the mean square MSE of the residual curvature is

$$
\mathrm{MSE}_{2}=\frac{1}{15} \cdot \sum_{i=0}^{14}\left(\Delta_{i}\right)^{2}=4.35 \times 10^{-7}
$$

The mean square of the residuals is 0 in the range of $10^{-6}$, which meets the required accuracy requirements. At this point, the curvature data after fitting the springback are the exponential function curve type. The obtained parameters are $a=1$ and $b=0.03$. At this point, the iteration ends, and the continuous curvature free bending forming parts are obtained by correcting the curvature $K$.

3.2.3. Iterative Result of Quadratic Function-304. 304 stainless steel sheet is $2 \mathrm{~mm}$ thick, and objective curve function equation is $y=0.001 x^{2}+0.002 x$. Iterative results of the quadratic function-304 model are shown in Figure 4; the expected value can be achieved by two iterative compensation for curvature $K$.

For the first iteration model, the curvature value of each data point decreases after rebound, and the $\Delta$ value is in the neighborhood of 0.0016 . After calculation, the mean square MSE of the residual curvature is

$$
\mathrm{MSE}_{1}=\frac{1}{15} \sum_{i=0}^{14}\left(\Delta_{i}\right)^{2}=2.56 \times 10^{-6}
$$

In the second iteration, the equation of compensation curve is expressed as $y=0.0019 x^{2}+0.0038 x$, error value after springback $\Delta$ in the right neighborhood of 0 . After calculation, the mean square MSE of the residual curvature is

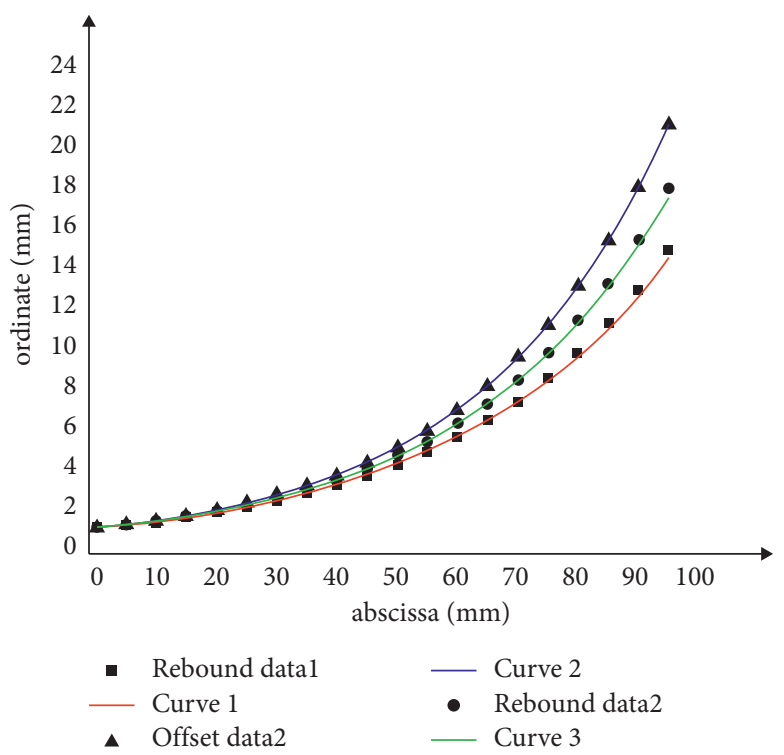

FIgURE 3: Exponential function-ST12 iteration results.

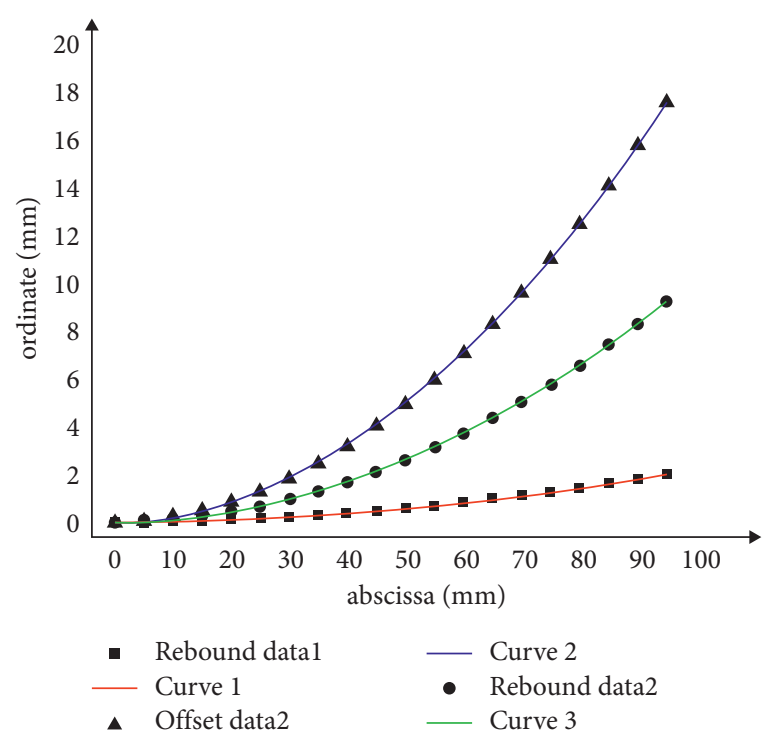

Figure 4: Polynomial function-304 iteration result.

$$
\mathrm{MSE}_{2}=\frac{1}{15} \sum_{i=0}^{14}\left(\Delta_{i}\right)^{2}=7.6 \times 10^{-8} .
$$

The mean square of the residuals is 0 in the range of $10^{-6}$, which meets the required accuracy requirements. At this point, the curvature data after fitting the springback is the quadratic function curve type. The obtained parameters are $a=0.001$ and $b=0.002, c=0$. At this point, the iteration ends, and the continuous curvature free bending forming parts are obtained by correcting the curvature $K$.

3.2.4. Index Function-304 Iteration Result. Iterative results of the exponential function-304 model are shown in Figure 5; the expected value can be reached after two iterative compensation of curvature $K$. 
For the first iteration model, the $\Delta$ values of each data point after unloading are in the neighborhood of 0.0009 . After calculation, the mean square MSE of the residual curvature is

$$
\mathrm{MSE}_{1}=\frac{1}{15} \cdot \sum_{i=0}^{14}\left(\Delta_{i}\right)^{2}=9.07 \times 10^{-7}
$$

In the second iteration, the equation of compensation curve is expressed as $y=e^{0.024 x}$, curvature error after springback decreases, and the $\Delta$ value is in the neighborhood of 0 . After calculation, the mean square MSE of the residual curvature is

$$
\mathrm{NSE}_{2}=\frac{1}{15} \cdot \sum_{i=0}^{14}\left(\Delta_{i}\right)^{2}=2.44 \times 10^{-7}
$$

The mean square of the residuals is 0 in the range of $10^{-6}$, which meets the required accuracy requirements. At this point, the curvature data after fitting the springback is the exponential function curve type. The obtained parameters are $a=1$ and $b=0.02$. At this point, the iteration ends, and the continuous curvature free bending forming parts are obtained by correcting the curvature $K$.

\section{Simulation Analysis of the Continuous Curvature Plane Bending Process}

Two materials are selected for the numerical simulation analysis in this article, namely, ST12 and 304, which are commonly used materials for sheets. The relevant mechanical parameters can be found in the "Material Performance Data Handbook for Mechanical Engineering" [16].

In this article, the finite element software Abaqus is used to simulate the bending springback. Establish a two-dimensional finite element model, the mold is represented by a two-dimensional curve, create a reference point, and the type is set to discrete rigid body; the sheet is represented by a two-dimensional plane, and the type is set as a deformable body. The mechanical properties of sheet metal are obtained by the tensile test.

4.1. Analysis of ST12 Simulation Results of Quadric Function. The free bending simulation is carried out on the model with material type ST12 and objective function $y=0.001 x^{2}+0.002 x+0$. For the first bending, the upper and lower model surface curve equations are expressed as $y=0.001 x^{2}+0.001 x+c$, where $c$ 's values are determined when the components are assembled.

After completing the position and displacement loading control, the deformation of the sheet metal at the end of loading is shown in Figure 6. The figure shows that when the sheet metal is loaded, the stress value is between 1.34 and $140.59 \mathrm{MPa}$. According to the experimental results, ST12, the stress-strain relationship of the material, plastic deformation occurs in this interval. The partial grid of data acquisition of sheet metal was not distorted.

After the bending loading is finished, the mold is removed, so that the sheet material can rebound freely. The sheet metal after springback is analyzed, and the deformation of the sheet metal after springback is shown in Figure 7.

At this point, the stress value of the sheet metal decreases after springback, and its value is between 0.19 and 86.95 $\mathrm{MPa}$, and all meshes are not distorted. The second bending loading simulation is carried out, and the profile curve is the profile curve after the curvature correction is compensated. Repeating the above operation, the die profile curve is obtained according to the theoretical part. The deformation of the forming parts after the first simulated bending rebound after the second loading and rebound is shown in the following two pictures.

Figure 8 shows that after iterative compensation of curvature, the stress value of sheet metal is between 9.55 and 144.61 MPa during the second bending loading. The partial grid of data acquisition was not distorted during loading.

After the second bending loading is finished, the mold is also unloaded, so that the sheet material can rebound freely. The analysis of the sheet metal after springback, from Figure 9 shows that the stress value of the sheet metal after springback is between $5.17107 .79 \mathrm{MPa}$, and all meshes are not distorted. The results show that the deformation of the sheet is good.

Because the simulation results can not be used as the result values of the specific calculation, but can only represent the change of the trend. Therefore, in the simulation results of the free bending process of sheet metal, this study only compares the trend of the neutral layer curves before and after springback and before iterative compensation.

The simulation results of the quadratic function-ST12 model are analyzed and processed; the origin is used to make a broken line diagram of each group of data corresponding to the target curve, the two rebound curves of sheet metal, and the curve of compensation profile. The simulation results of this model are compared as shown in Figure 10.

From the simulation results above, we can see that the shape of sheet metal obtained from the shape curve after the second iteration compensation is closer to the target shape. As the number of iterations increases, the error between the resulting value and the expected target value becomes smaller and smaller, indicating that the proposed iterative compensation mechanism for curvature is feasible in the quadratic function-ST12 model.

4.2. Exponential Function-ST12 Simulation Results Analysis. The free bending simulation is carried out on the model with material type ST12 and objective function $y=e^{0.03 x}$. For the first bending, the upper and lower model surface curve equations are expressed as $y=e^{0.03 x}+c$, where $c$ 's values are determined when the components are assembled, and in other processes, analogy quadratic function analysis results of the ST12 model. During the two bending loading and springback, there was no misalignment, distortion, or fracture of sheet metal. The neutral layer data of sheet metal after two springbacks were extracted, and trend line was added to compare with profile curve and target shape. The contrast results are shown in Figure 11. 


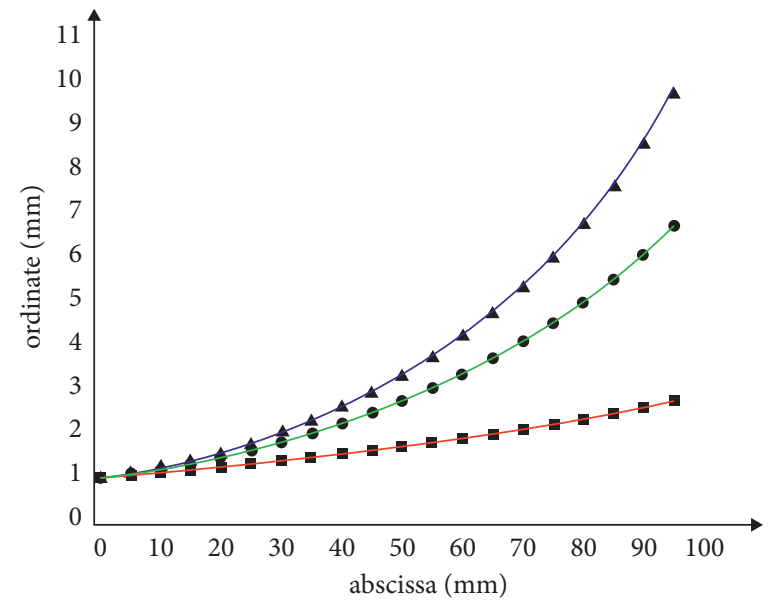

- Rebound data1

- Offset data2

- Rebound data2

Figure 5: Index function-304 iteration result.

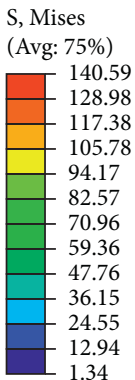

FIGURE 6: Sheet deformation diagram after the first loading.

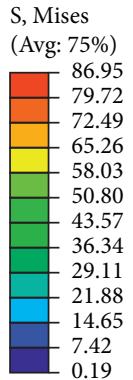

Figure 7: Sheet deformation diagram after the first springback.

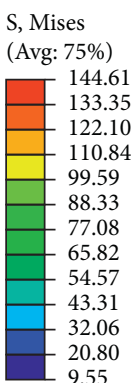

FIgURE 8: Sheet deformation diagram after the second loading. 


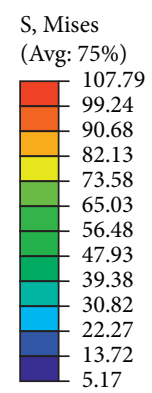

FIGURE 9: Sheet deformation diagram after the second springback.

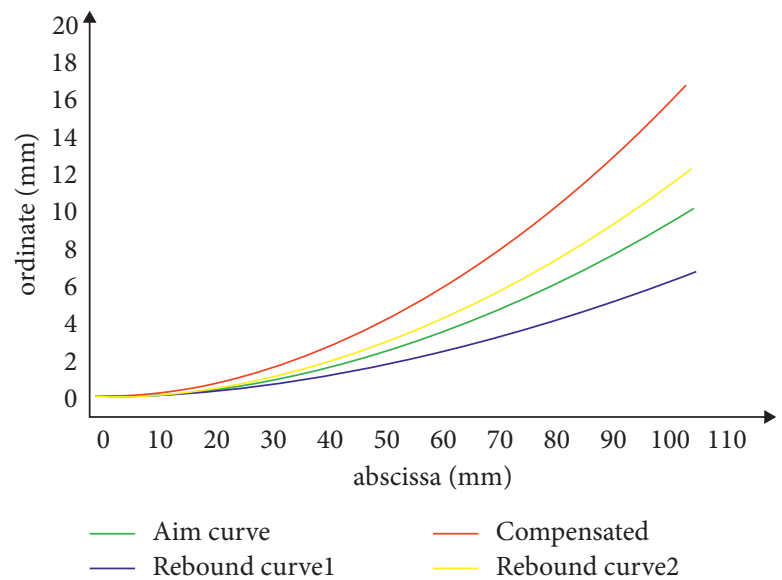

Figure 10: Dual function-ST12 simulation results.

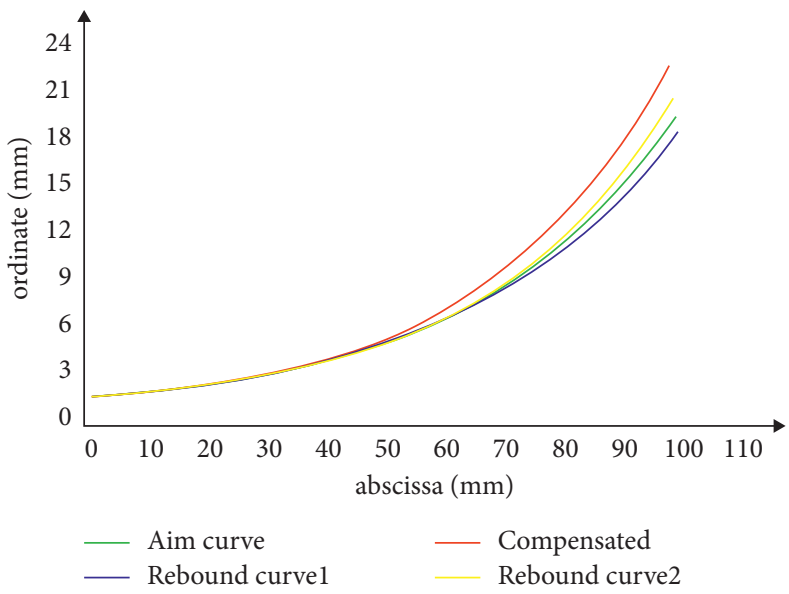

Figure 11: Exponential function-ST12 simulation results.

Compared with the model of quadratic function, the deformation of the exponential function model is larger. After each loading and rebound, the stress value of sheet metal is higher than the simulation result value of quadratic function. Comparing the two models of ST12 materials, we can see that for the same material, different function types do not affect the application of the iterative compensation mechanism. For both functional models, the shape of the forming parts obtained from the profile curve after the second iteration compensation is closer to the target shape. As the number of iterations increases, the error between the obtained result value and the expected target value is becoming smaller and smaller. 
4.3. Analysis of Simulation Results of Quadric Function-304. The simulation of the quadratic function-304 finite element model is analogous to the analysis result of the quadratic function-ST12 model. The process will not be repeated.

The simulation results are analyzed: during the two bending loading and springback, there is no dislocation, distortion, or fracture of sheet metal mesh. The neutral layer data of sheet metal after two springbacks were extracted, and trend line was added to compare with profile curve and target shape. The contrast results are shown in Figure 12.

Compare the simulation results of the quadratic function-304 model with those of the quadratic function-ST12 model and analyze the application of the curvature iterative compensation mechanism under different material models of the same function type. The shape of the target to be achieved is the same, and the amount of bending rebound of 304 sheet metal after springback is larger. Hence, the iterative compensation value for curvature is also relatively large, and the variation of the compensation profile curve is also larger than that of ST12 material. This result is related to material properties. However, for the model of two materials, the shape of the sheet material after rebound from the profile curve after the second iteration compensation is closer to the target shape. As the number of iterations increases, the error between the obtained result value and the expected target value is becoming smaller and smaller.

\subsection{Analysis of Simulation Results of Exponential Function-} 304. The simulation of the exponential function-304 finite element model is analogous to the analysis result of the exponential function-ST12 model. The objective curve and iterative compensation curve of exponential function are obtained by the theoretical model.

The simulation results are analyzed; during the two bending loading and springback, the sheet metal is formed evenly without distortion or penetration. The neutral layer data of sheet metal after two springbacks are analyzed, and the comparison results are shown in Figure 13. In the analysis of the finite element simulation results of the exponential function-304 model, comparing the result data with the simulation results of the other three models, we can get the contrast conclusions in three cases: different function equations, different materials, and different deformation quantities.

Compared with the exponential function-ST12 model, the deformation of the 304-material exponential model is smaller. Comparing the two models, we can see that for the same material, the effect of different deformation of the same function type on the application of the iterative compensation mechanism. From the previous, we have known that different materials of the same function type do not affect the application of the curvature iterative compensation mechanism. For the comparison of exponential function-304 and exponential function-ST12, we can analyze the influence of different deformation amount on the iterative compensation mechanism in the continuous curvature plane bending process. For both models, the sheet shape obtained from the profile curve after the second iteration compensation is closer to the target shape. Moreover, with the increase of the number of iterations, the error between the obtained result value and the expected target value is becoming smaller and smaller.

\section{Experimental Analysis of Continuous Curvature Plane Bending}

This section will verify the feasibility of the curvature iterative compensation mechanism based on four mathematical models from the perspective of experimental analysis.

It can be proved by numerical calculation and graphical simulation that the upper and lower surface curves can be used to replace the neutral layer curve and meet the accuracy requirements. Therefore, in the experiment, this study uses the upper surface curve to replace the neutral layer curve.

5.1. Experimental Scheme. The surface curve equation of the bending die is determined by the target shape of the sheet metal. Bend loading of the sheet metal and measure the actual shape of the sheet metal after unloading the springback. The least square method is used to fit the measurement data to obtain the curve equation of the forming parts. Using the curvature iterative compensation theory proposed in this study, the profile curvature of the bending die at the initial loading is compensated, and the profile curve of the forming die is corrected. The compensated bending die is used to bend and load the sheet material after the last rebound to determine whether the shape of the sheet material meets the target shape after the rebound. If satisfied, the iterative compensation process ends. If it is not satisfied, the curvature iterative compensation mechanism proposed in this study is used to correct the curve of curved die. Repeat this operation until the shape of the sheet material after rebound meets the target shape, and the curvature of each point approximates the target value within the error allowable range. Finally, the experimental results are compared with the theoretical and simulation results to verify the reliability of the iterative mechanism in the continuous curvature plane bending rebound problem.

5.2. Experimental Mold. Check the strength and load force required for bending experiment of ST12 and 304 materials; the material of the experimental mold is steel 45 . Steel no. 45 is a common material for stamping die with high strength and good machinability. Considering this experiment is to explore the confirmatory experiment, the forming parts do not carry out mass production, so the mold does not need to be quenched.

The die holder part contains three parts: connecting plate, upper template, and lower die holder. The connecting plate is mainly used to connect the mold with the multifunction electronic pull-torsion tester. The connecting plate is fixed with the upper template and the upper mold, and the inner hexagonal screw is used between the lower mold and the lower mold seat. Four guide sleeves and guide postmounting holes are symmetrically distributed around the upper template and lower die seat for installing guide positioning parts. 


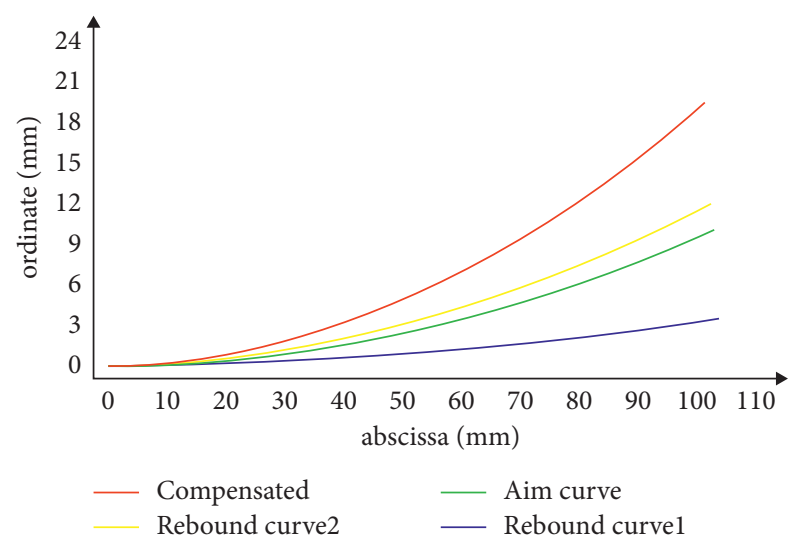

FIgURE 12: Simulation results of quadratic function-304.

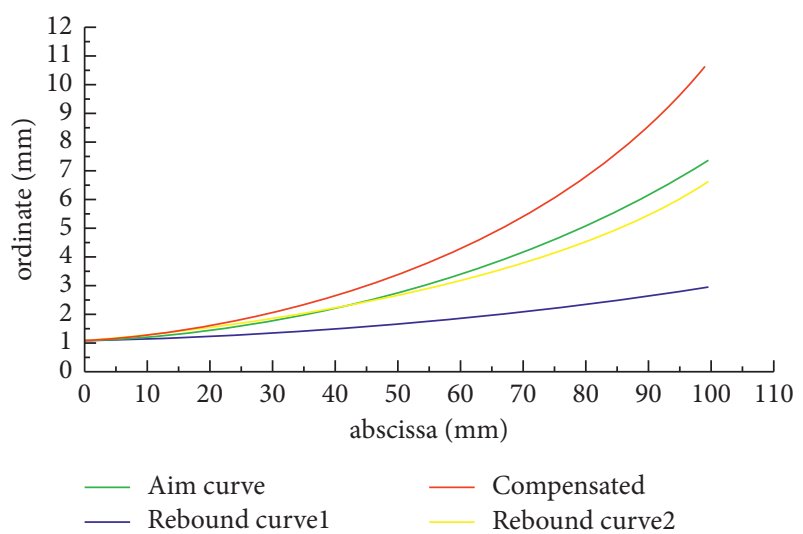

FIGURE 13: Exponential function-304 simulation results.

The guide part is a vital part in the design of stamping die. In the plane bending experiment of sheet metal, the guide mechanism of guide column-guide sleeve is adopted, and 4 equal straight guide columns are arranged symmetrically. Guide column and guide sleeve are selected standard lead type.

In the continuous curvature plane bending experiment of the two materials, the forming die mainly includes three parts: upper die, lower die, and press block. The main forming part of the upper die is the profile part, and the profile curve is obtained from the theory obtained in the first two chapters of this study. The purpose of the pressing block is to fix the sheet metal during bending and unloading to ensure the normal operation of the experiment. The press block is fixed to the lower die by screws, which acts as a fixing plate material.

Taking the exponential function-304 model as an example, the forming die required for the first continuous curvature plane bending experiment of the specimen is shown in Figure 14.

\subsection{Experimental Parametric Control and Process Analysis.} The equipment for the bending experiment is the link real pull-torsion tester (Figure 15), which contains two parts: the main engine and the pc control end. Experiment type is

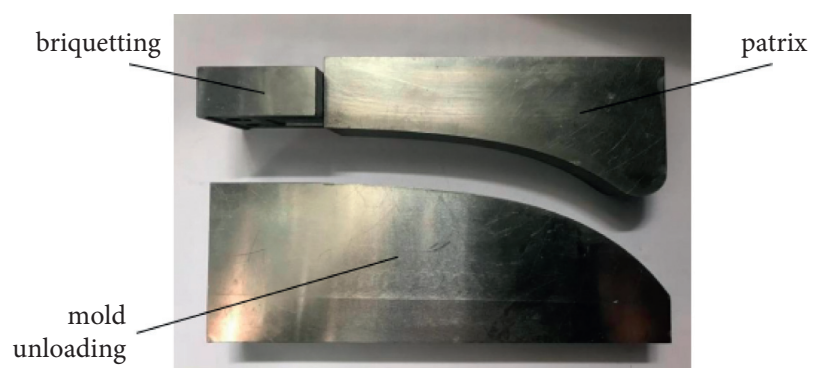

FIgure 14: Physical photo of first bending die for the exponential function-304 model.

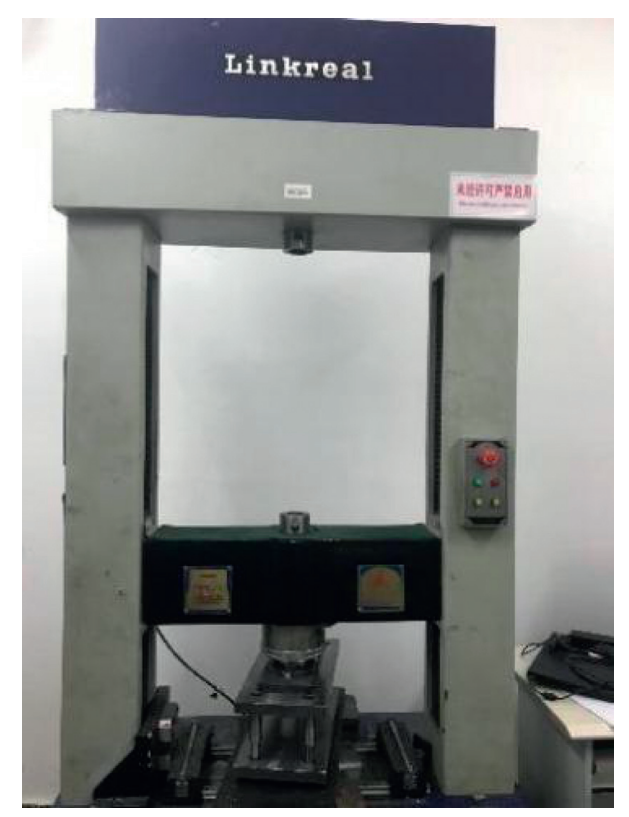

Figure 15: Pull-torsion tester.

chosen as compression, and the maximum load of equipment is $150 \mathrm{kn}$ lenleadertwodots. Pull and twist testing machine and mold installation is shown in Figure 16. After the mold is installed, turn on the power supply and turn on the $\mathrm{pc}$ end control software.

Before the experiment begins, zero the device and create a working directory and file. The free bending experiment of sheet metal is completed by controlling the displacement. Load at a speed of $10 \mathrm{~mm} / \mathrm{min}$ before the upper die touches the sheet. After the upper die touches the sheet, the loading speed decreases to $1 \mathrm{~mm} / \mathrm{min}$, and then, the speed is adjusted to a minimum of $0.1 \mathrm{~mm} / \mathrm{min}$. When the die is close, the displacement-force curve of the output of the equipment is monitored in real time during the experiment to ensure that the loading amount does not exceed the equipment load. After the loading process is completed, control the pulltorsion tester press head up and unload the sheet metal. Because the experiment of the same condition was repeated in three groups, the opening height was measured by the suitable replacement specimen.

The data after rebound of sheet metal are processed to obtain the actual rebound curve of sheet metal. Then, the curvature iterative compensation theory is applied to 


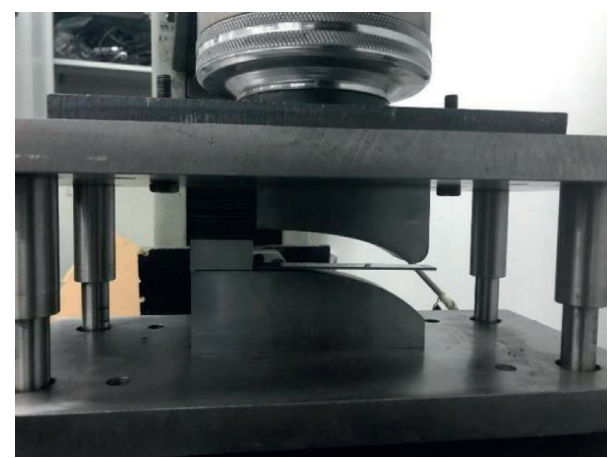

FIgURE 16: Mold installation chart.

calculate the compensation profile curve. Replace the iterative compensation model to carry out the loadingunloading experiment on the sheet metal at the end of the previous experiment. Finally, the experiment is finished after the sheet metal shape reaches the target shape.

5.4. Analysis of Experimental Results. The analysis of the forming parts obtained from the continuous curvature plane bending experiment also includes two parts. At the end of each loading-unloading process, observe the sheet metal forming. To observe whether extrusion thinning occurs in sheet metal, if so, the experiment under this condition needs to be carried out again. When the sheet metal forming condition is good, the shape of sheet metal is further analyzed. Use a three-coordinate measuring instrument to take points on the upper surface of the test piece to obtain the coordinate points of the shape function of the upper surface, fitting the equation of sheet metal curve after springback, and judging whether it needs iterative compensation.

5.4.1. Experimental Results Loaded with Target Shape. In the bending experiment, the sheet metal of the four models has elastic-plastic deformation. After unloading, the elastic deformation of the sheet metal disappears and the plastic deformation is retained. The shape surface shapes with the target shape as the mold. After the loading-unloading process is finished, the sheet material deformation occurs after the springback of the four models as shown in Figures 17-20 (formwork after first bending rebound). In order to compare the effect clearly, put the springback sheet forming parts together with the straight plate to observe the deformation amount.

Observing the four forming parts, the thickness of the sheet material is uniform after deformation, and no extrusion thinning or fracture occurs. It shows that the experimental scheme of free bending forming of sheet metal is feasible and the experimental operation is correct.

Seris3000i coordinate information of the first forming sheet was collected by using the three-coordinate measuring instrument, and the starting and ending points of the strip marking part and the terminating position of the sheet metal experimental part were recorded. Each forming part is measured separately, and the measurement results are in data points for $3 \mathrm{D}$ space. Each data point is projected onto the initially set datum surface to obtain the plane coordinate values of each measurement point.

The coordinate information of the four models after springback is fitted to the curve, and the fitting results are given in Table 4.

\subsubsection{Experimental Results of Iterative Compensation.} After obtaining the curve equation of the first forming part, according to the curvature limit condition and the arc length limit condition, the curvature is modified to obtain the compensated profile curve. The curve equations of the four models after compensating the curvature are given in Table 5.

After determining the profile curve of the compensation die, the iterative compensation die is processed by the EDM cutting machine. The profile curve is polished to ensure the continuous smoothness of the profile curve. Repeat the experiment, a second bending experiment for the sheet material after the previous loading-unloading.

The initial straight plate, the specimen after the first bending rebound, and the left end of the specimen after the second compensation bending are fixed, and the deformation of the sheet material is observed together. From top to bottom are the specimens after the second bending rebound, the specimens after the first bending rebound, and the straight plate specimens without bending.

After the compensation experiment, the sheet metal forming parts are obtained as shown in Figures 21-24 (formwork after second bending rebound).

Similarly, no extrusion thinning and fracture were observed in the specimen because the selected profile curve is the target shape at the first loading. So, the sheet material after each rebound can be fitted with the first profile curve. If it can be fully fitted, it shows that the iterative compensation process can be ended and the experimental value has reached the expected value.

Coordinate measurement of the specimen is obtained from the compensation experiment, fitting the function curves of the related types to the measured data and judging whether the iterative process ends from the coefficient value of the expression of the function equation. The second-class results of the data fitting function curve are given in Table 6. 


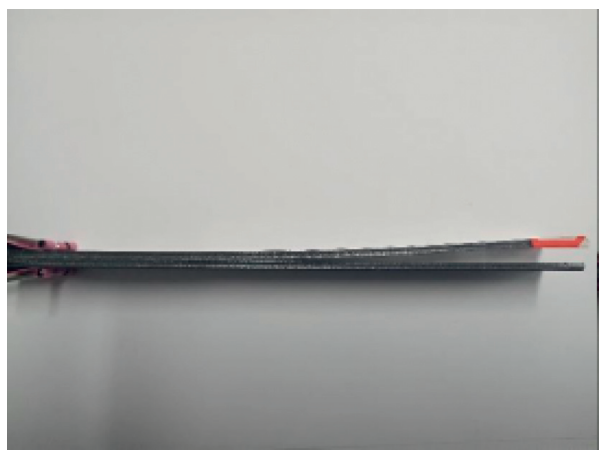

Figure 17: Quadric function-ST12.

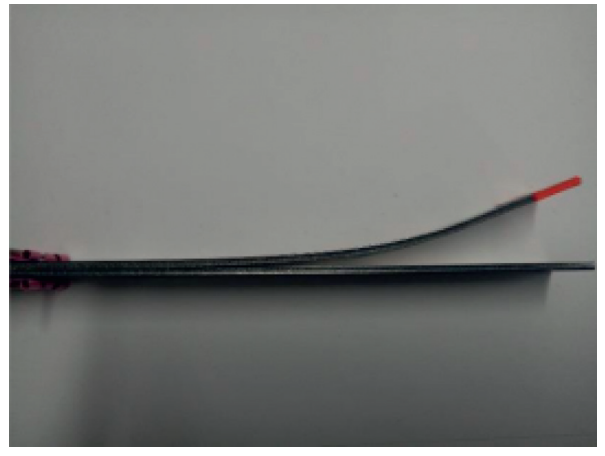

FIgURE 18: Exponential function-ST12.

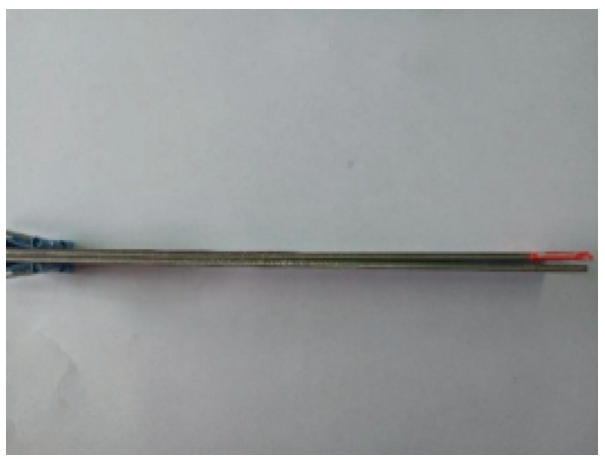

FIGURE 19: Quadric function-304.

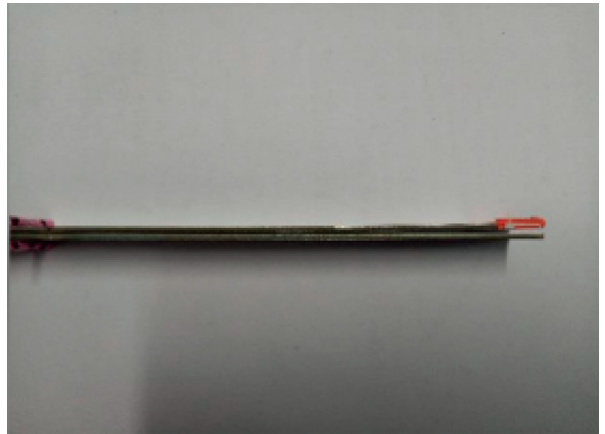

FIgURe 20: Exponential function-ST304. 
TABLE 4: Fitting results of four model sheet metal curves after the first bending experiment.

\begin{tabular}{lcccc}
\hline Model & $a$ & $b$ & $c$ & Whether close to expections \\
\hline Quadratic function-ST12 & 0.0004 & 0.002 & 0.0006 & No \\
Exponential function-ST12 & 1 & 0.0032 & - & No \\
Quadratic function-304 & 0.0001 & 0.002 & 0.0009 & No \\
Exponential function-304 & 1 & 0.024 & - & No \\
\hline
\end{tabular}

TABLE 5: The modified surface curve equation of four models.

\begin{tabular}{lcccc}
\hline Model & $a$ & $b$ & $c$ & Maturity \\
\hline Quadratic function-ST12 & 0.0017 & 0.0033 & 0.0006 & 1 \\
Exponential function-ST12 & 1 & 0.032 & - & 1 \\
Quadratic function-304 & 0.002 & 0.004 & 0.001 & 1 \\
Exponential function-304 & 1 & 0.024 & - & 1 \\
\hline
\end{tabular}

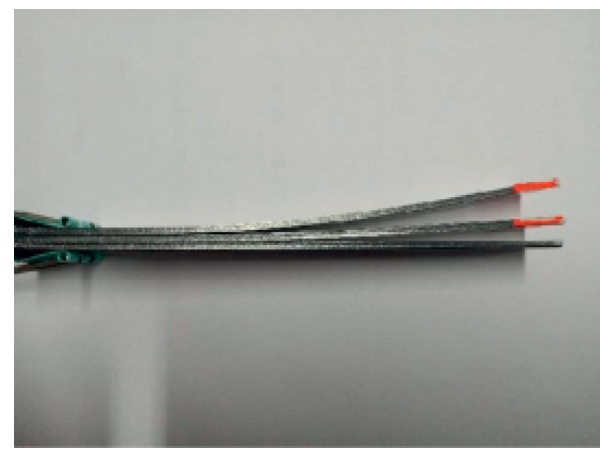

FIgURE 21: Quadric function-ST12.

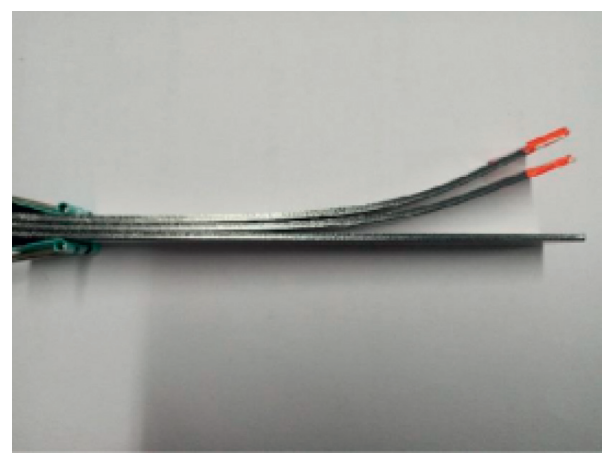

FIgURE 22: Exponential function-ST12.

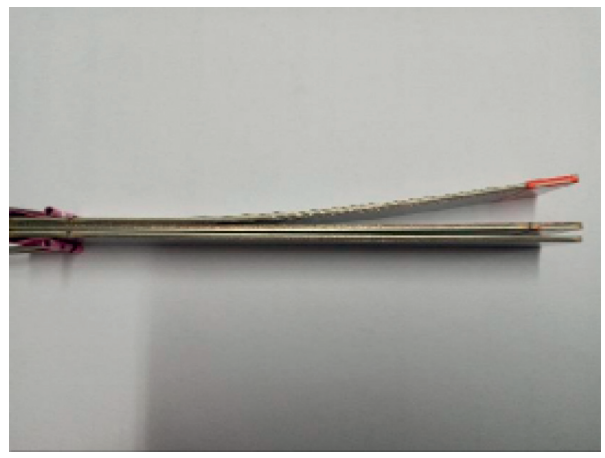

Figure 23: Quadric function-304. 


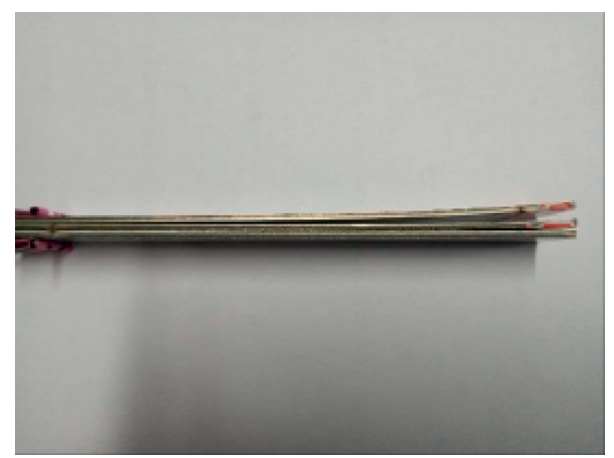

FIGURE 24: Exponential function-ST304.

TABLE 6: Fitting results of four models after compensation experiment.

\begin{tabular}{lccc}
\hline Model & $a$ & $b$ & Whether close to expections \\
\hline Quadratic function-ST12 & 0.001 & 0.002 & Yes \\
Exponential function-ST12 & 1 & 0.03 & Yes \\
Quadratic function-304 & 0.001 & 0.002 & Yes \\
Exponential function-304 & 1 & 0.02 & Yes \\
\hline
\end{tabular}

From the above figure, we can see that after the compensation experiment, the curve fitting result after the rebound of sheet metal reaches the expected value on the parameter value. Judging the end of the iterative compensation process of the four models, the target specimen can be obtained by bending loading of the compensation die.

\section{Conclusion}

(1) In the free bending process of plate plane, curvature is feasible as an iterative parameter of springback compensation control. A new method to analyze the rebound problem of continuous curvature plane bending is proposed, that is, the curvature iterative compensation mechanism.

(2) The iterative compensation mechanism of the four models is expressed, and the feasibility of the proposed iterative method is verified from the perspective of theoretical calculation. By comparing the models, it is shown that within a certain range, the iterative compensation mechanism of curvature correction does not depend on the material type and function type.

(3) The finite element software simulation shows that the neutral layer curves of four models converge to the target shape with the increase of iteration times and trend to verify the correctness of the proposed theory

(4) The experimental results show that the shape of sheet metal can converge to the target value after two bending loads through iterative compensation of curvature, which has high convergence speed and control accuracy. The successful application of the curvature iterative compensation mechanism in four models proves its feasibility in engineering applications.
(5) In this study, the feasibility of the iterative compensation mechanism of curvature in the continuous curvature plane bending process is verified by the combination of theoretical analysis, finite element simulation, and experiment, and the characteristics of this method are not limited to steel types, and function types are revealed.

\section{Data Availability}

The data used to support the findings of this study are included within the article.

\section{Conflicts of Interest}

The authors declare that there are no conflicts of interest.

\section{Acknowledgments}

This work was funded and supported by the Natural Science Foundation of Hebei Province in China, Ministry of Industry and Information Technology of the People's Republic of China, and the Development and Application of Key Equipment for Sheet Metal Forming of High Strength and Lightweight Alloy-Major Special Project of Science and Technology (2018Z×04007002-004-1).

\section{References}

[1] L. Yang, Stamping Processes and Dies Design, pp. 2-18, Xidian University Press, Xi'an, China, 2018.

[2] R. Hill, The Mathematical Theory of Plasticity, Oxford University Press, London, UK, 1950.

[3] F. J. Gardiner, "The springback of metals," Transactions of the ASME, vol. 79, pp. 1-9, 1957.

[4] C. A. Queen and R. J. DeAngelis, "Elastic springback and residual stresses in sheet metal formed by bending," ASM Transactions, vol. 61, pp. 757-768, 1968. 
[5] X. A. Yang and F. Ruan, "A die design method for springback compensation based on displacement adjustment," International Journal of Mechanical Sciences, vol. 53, no. 5, 2011.

[6] K. J. Weinmann, A. H. Rossenberger, and L. R. Sanchez, "The Bauschinger effect of sheet metal under cyclic reverse pure bending]," Annals of CIRP, vol. 37, no. 1, pp. 2-5, 1998.

[7] J. Zhao, J. Yin, R. Ma, and L. Ma, "Springback equation of small curvature plane bending," Science in China: Technology Science, vol. 54, no. 9, pp. 2386-2396, 2011.

[8] S. W. Lee and D. Y. Yang, "An assessment of numerical parameters influencing springback in explicit finite element analysis of sheet metal forming process," Journal of Materials Processing Technology, vol. 80-81, no. 89, pp. 60-67, 1998.

[9] J. L. Song, D. J. Hu, and Z. L. Chang, "Study on the finite element numerical simulation of sheet metal forming," Acta Metallurgica Sinica, vol. 17, no. 3, pp. 299-302, 2004.

[10] B. Ji and W. J. Chen, "A new analytical solution of pure bending beam in couple stress elasto-plasticity: theory and applications," International Journal of Solids and Structures, vol. 6 , no. 6, 2010.

[11] J. T. Gau and G. Kinzel, "A new model for springback prediction in which the Bauschinger effect is considered," International Journal of Mechanical Sciences, vol. 43, no. 8, 2001.

[12] Z. Zhang, R. Ma, C. Wang, and J. Zhao, "Research on Springback control in stretch bending based on iterative compensation method," Mathematical Problems in Engineering, vol. 2019, Article ID 2025717, 2019.

[13] R. Ma, C. Wang, and J. Zhao, "An iterative compensation algorithm for springback control in plane deformation and its application," Chinese Journal of Mechanical Engineering, vol. 32, no. 1, p. 28, 2019.

[14] R. H. Wagoner and M. Li, "Simulation of springback: through-thickness integration," International Journal of Plasticity, vol. 23, no. 3, 2007.

[15] W. L. Xu, C. H. Ma, C. H. Li, and W. J. Feng, "Sensitive factors in springback simulation for sheet metal forming," Journal of Materials Processing Technology, vol. 151, no. 1-3, 2004.

[16] Editorial Board of Mechanical Engineering Material Performance Data Handbook. Material Performance Data Handbook for Mechanical Engineering, pp. 21-32, Mechanical Engineering Press, Beijing, China, 1995. 